\title{
L'arte della memoria. Antiche esperienze e moderne suggestioni.
}

\author{
Lina Bolzoni
}

È molto bello e utile imparare, da ragazzi, poesia a memoria, diceva Calvino in una straordinaria intervista che la televisione-strumento per eccellenza di formazione e controllo del ricordo, oltre che di manipolazione del tempoha trasmesso poco dopo la sua morte. Calvino ci riproponeva così l'estrema propaggine scolastica, ormai quasi in disuso, di una pratica che l'Occidente ha coltivato per secoli, almeno fino al Settecento: la mnemotecnica, o arte della memoria (cfr. Rossi; Yates; Bolzoni, "Il gioco"; Carruthers; AA. VV., Mnemosyne; Gedächnistkunst).

Oggi l'arte della memoria ci appare, per molti aspetti, come un oggetto estraneo, un fossile giunto da un mondo scomparso. Ci sembra davvero incredibile che, per secoli, gli uomini abbiano impiegato tempo ed energie, abbiano praticato e insegnato tecniche per aumentare le capacità naturali di ricordare. Viviamo infatti in un tempo in cui il problema di ricordare a memoria le cose ha perso senso, consistenza, valore: abbiamo delegato alla scrittura, ai libri e a strumenti tecnologici sempre più sofisticati, il compito di conservare parole, immagini, suoni, conoscenze. Viviamo d'altra parte in uno spazio in cui, a ritmi del tutto sconosciuti al passato, le immagini si muovono, si trasformano, si frantumano, si consumano rapidamente. Si è enormemente dilatato quel cambiamento della percezione visiva che Walter Benjamin aveva collegato alla nascita della moderna metropoli, con la sua folla in movimento (104).

Rispetto alla nostra esperienza, dunque, l'arte della memoria richiede in primo luogo di essere riconosciuta nella sua diversità. Sparsi sui polverosi scaffali delle antiche biblioteche europee, numerosi trattati di arte della memoria che i secoli ci hanno tramandato richiamano infatti la nostra attenzione anche su ciò che sta oltre il testo: complessi scenari si aprono se si capisce che i trattati sono solo la punta di un iceberg, e che i loro precetti-spesso aridi e ripetitivi-sono solo la scenografia di uno spettacolo che si sviluppa a più dimensioni.

L'arte della memoria, infatti, non è soltanto una disciplina, o una tecnica specifica e autonoma: essa interagisce con pratiche e esperienze diverse, che riguardano la poesia e la pittura, la mistica e la scienza, la filosofia e la retorica. 
L'arte della memoria, inoltre, ci dice molto anche sul soggetto che la pratica. Se noi ci interroghiamo su chi è l'uomo che i nostri trattati insieme presuppongono e costruiscono, vediamo delinearsi, attraverso i secoli, un'immagine per molti aspetti costante. Ed è appunto questa immagine di uomo a presentarsi a noi con caratteri che sono insieme, in un modo un po' inquietante, estranei e familiari.

Cosa ha significato, infatti, praticare l'arte della memoria? Ha significato impegnarsi in lunghi, pazienti esercizi di conoscenza, di controllo, di dilatazione delle proprie qualità psichiche: della memoria in primo luogo, ma anche della immaginazione e della sensibilità, del modo di reagire alle immagini, alle associazioni, ai giochi di parole.

Almeno dall'età in cui si diffonde la scrittura, inoltre, l'arte della memoria ha insegnato a plasmare la propria mente, a scandirla entro spazi ordinati, a costruire elaborate architetture interiori. Come le lettere dell'alfabeto frantumano il flusso del discorso orale, lo sottraggono al tempo vivente della comunicazione interpersonale, ma proprio a questo prezzo lo fanno vivere nello spazio e nel tempo, ${ }^{1}$ così agisce l'arte della memoria nei confronti del magma caotico delle immagini mentali: lo studia, lo analizza, cerca di ricondurre a leggi il gioco affascinante delle associazioni, cerca di capire-e di riprodurre-la logica per cui un'immagine ne richiama (o ne nasconde) un'altra. Le immagini appaiono allora simili alle lettere dell'alfabeto: segni che bloccano, e nello stesso tempo fanno rivivere il flusso dei ricordi; immagini artificiali, capaci però di far sprigionare di nuovo da sé, al momento opportuno, quell'esperienza vitale cui hanno dato maschera e forma.

Ed è appunto su questa paziente costruzione di immagini interiori che l'arte della memoria ha appuntato la sua attenzione. Essa ha insegnato a costruire imagines agentes, il che significa in primo luogo immagini capaci di svolgere un'azione, di colpire, immagini che condensano in sé emozioni e conoscenze, in un intreccio tale che l'impatto delle prime faccia scattare la catena delle seconde. E d'altra parte imagines agentes significa anche immagini capaci di rappresentare una parte. Esse rinviano cioè a quella dimensione teatrale che è così importante nella pratica mnemonica, ad esempio nella tecnica per cui, per ricordare concetti astratti, o parole di una lingua straniera, si chiede loro di raccontare una storia, facendo scaturire dalle parole, con appositi procedimenti, dei personaggi che si confrontano in un dialogo, in una scena; ma anche dimensione teatrale in un senso più generale e più profondo. Cicerone scrive infatti (De oratore 2.88 .359 ) che bisogna collocare delle maschere teatrali (personae) sopra i concetti, così da trasformarli in immagini attive nella nostra memoria, in imagines agentes appunto.

L'arte della memoria vuole plasmare sia la costruzione che la recezione delle immagini, vuole assicurarsi che ci sia un pieno rispecchiamento fra la prima e la seconda. La cura nel controllare i modi in cui le immagini nascono e si sviluppano dalla catena delle associazioni corrisponde alla preoccupazione di condizionare i modi, i tempi, i contenuti della fruizione delle immagini 
stesse. In altri termini l'arte della memoria è simile a un regista ambizioso, che considera la capacità di prevedere le reazioni del pubblico come un elemento costitutivo dello spettacolo. Il teatro della memoria di Giulio Camillo è del resto anche un teatro rovesciato, in cui si ribalta il rapporto tradizionale fra spettatori e spettacolo, tra chi vede e ciò che è visto: lo spettatore è sulla scena, e lo spettacolo si dispone ordinatamente sulle gradinate.

Perché lo spettacolo della memoria si metta in moto e funzioni, bisogna che l'occhio della mente percorra le immagini in modo lento, ordinato, analitico. L'arte della memoria richiede una visualità molto lontana dalla nostra, una visualità capace di far sprigionare dall'immagine tutti i messaggi di cui è stata investita, una visualita, quindi, attenta a cogliere tutti gli aspetti del rapporto fra l'ordine, lo spazio, l'immagine stessa, oltre a ripercorrere il gioco di relazioni fra parti e tutto, fra pluralità e unità. Proprio per queste sue caratteristiche, del resto, l'immagine di memoria può interagire con la costruzione dell'allegoria; come si sia storicamente configurato il rapporto fra le due pratiche, in un gioco di continuità e di profonde differenze, è un affascinante problema aperto.

È nei vasti territori della mente, dunque, che l'arte della memoria costruisce i suoi "luoghi" e le sue immagini, ma questo non esclude affatto-in molti casi addirittura presuppone-che si crei un gioco di rinvii, di interazione, con luoghi e immagini esteriori, creati da pittori, architetti, scultori, oppure evocati dalle parole dei poeti e degli scrittori. Sappiamo molto-grazie agli studi di Paolo Rossi e di Frances Yates-sul modo in cui l'arte della memoria, attraverso i secoli, si è intrecciata con la storia della filosofia e della scienza, ma la questione di come l'arte della memoria ha interagito con la letteratura e le arti figurative costituisce tuttora, io credo, un affascinante capitolo aperto della storia culturale europea.

Le tecniche della memoria si sono dunque collocate, attraverso i secoli, in una zona che attraversa frontiere diverse: hanno cercato di controllare, si diceva, le vie che collegano l'interiorità e l'esteriorità; si sono esercitate nel tradurre le parole in immagini, e le immagini in parole. Giovan Battista Della Porta, ad esempio, consiglia a chi si avventura da principiante nei territori della mnemotecnica di esercitarsi nella costruzione e nella soluzione dei rebus (16).

Interessata alle ricche risorse che scaturiscono dalla visualizzazione del linguaggio, l'arte della memoria si è avventurata negli spazi che si aprono fra il significante e il significato: insofferente di rapporti univoci, ha sperimentato tutti gli effetti illusionistici e stranianti che nascono dalla frammentazione delle parole e dal gioco delle combinazioni.

Non a caso "gioco" è termine che ci viene più volte spontaneo di usare. Fra le immagini e gli strumenti dell'arte della memoria troviamo infatti la scacchiera del gioco degli scacchi, o mazzi di carte da gioco. I trattati di memoria indicano i precetti generali, danno il quadro delle procedure, ma invitano subito dopo alla sperimentazione, alla creazione individuale. Entro 
un quadro di possibilità date, ciascuno deve creare le proprie immagini, deve raccontarsi la propria storia. Modi di procedere cervellotici e accostamenti astrusi lasciano spesso il campo, nella costruzione delle immagini, a una forte carica di sensualità, a fantasie erotiche e sadiche. Il piacere e l'orrore, come teorizzano fin dalle origini i trattati, sono del resto molle psicologiche potenti, e l'arte della memoria è interessata soprattutto a che i suoi meccanismi funzionino, e ottengano dei risultati. L'ordine delle architetture mnemoniche fa così spesso da sfondo al disordine delle associazioni fantastiche; entro la razionale successione dei loci, trovano posto immagini miste, nate dalla proliferazione di accostamenti inconsueti.

La difficile impresa di coniugare insieme i contrari è del resto familiare a un'arte che studia i meccanismi naturali del ricordo e dell'oblio per poterli riprodurre artificialmente. L'arte della memoria viene così ad operare su di un terreno intermedio fra automatismo e consapevolezza, tra spontaneità e controllo.

Basata soprattutto sulla operatività delle immagini mentali, l'arte della memoria cerca di intervenire anche sul corpo, di condizionarlo in modo da facilitare il ricordo. Medicine, consigli di diete e di comportamenti, oltre che l'uso di vere e proprie droghe, si intrecciano così con la costruzione dei loci e delle imagines mentali. Praticando ancora un volta una zona di frontiera, l'arte della memoria cerca di agire entro quegli spazi-misteriosi ma essenziali-in cui il corpo e la psiche si incontrano e interagiscono.

Possono forse apparire da queste brevi annotazioni le ragioni per cui l'arte della memoria ci appare oggi in una luce di estraneità ma anche di inquietante familiarita. Lo abbiamo in un certo senso sperimentato attraverso la mostra intitolata: "La fabbrica del pensiero. Dall'arte della memoria alle neuroscienze". Per la prima volta le antiche immagini mnemotecniche sono uscite dal chiuso dei libri e dei manoscritti e hanno affrontato l'impatto con il grande pubblico, prima a Firenze, a Forte Belvedere, e poi a Parigi, negli spazi un po' fantascientifici della Villette. ${ }^{2}$

Per chi studia l'arte della memoria, inoltre, è veramente emozionante vederla riapparire-misconosciuta, ma ben provvista di tutti i suoi attrezzinelle pagine in cui il grande neurologo Alexander Lurija ci racconta delle tecniche usate da Shereshevski, un uomo che, nella Russia degli anni '30, si esibisce come mnemonista e fa i conti, nella vita quotidiana, con la sua capacità un po' angosciosa di ricordare tutto.

Ed è molto suggestivo anche il fatto che oggi il neuropsicologo Oliver Sacks incontri gli studi della Yates sull'arte della memoria nel momento in cui, dovendo curare persone che hanno gravi problemi psichici, non le riduce a casi clinici, ma cerca di ricostruire la loro storia personale, e soprattutto cerca di scoprire anche diversi modi di approccio al reale, la diversa "grammatica della fantasia" che la turba psichica pud mettere in moto (Sacks, parte 4).

L'arte della memoria, d'altra parte, rifa capolino qua e là anche nel campo 
della sperimentazione letteraria e figurativa del nostro tempo. I suoi antichi alfabeti figurati ricompaiono ad esempio nelle pagine di Massin, mescolandosi con i nuovi prodotti della contaminazione fra lettera e immagine da lui stesso creati; la presentazione di Queneau ci dà subito la chiave di questo recupero di materiale mnemotecnico: il moderno riproporsi del mito combinatorio e ludico nella creazione letteraria.

Il grande interesse che, nella critica letteraria degli anni ' 60 , hanno suscitato lo strutturalismo e la semiotica, ha inoltre riproposto-anche se naturalmente in chiave diversa-alcuni elementi costitutivi della tradizione dell'arte della memoria: l'idea, ad esempio, della combinatoria, della "macchina" narrativa, della possibilità cioè di ridurre la pluralità dei testi a una griglia topica, a una rete di combinazione fra componenti fisse. Suggestioni riconducibili all'arte della memoria ricompaiono così fra gli ingredienti usati da quegli autori in cui gli interessi per la teoria letteraria e la metodologia critica sono molto forti e hanno una ricaduta sulla stessa scrittura letteraria. Si pensi ad esempio a Italo Calvino, in particolare al Castello dei destini incrociati. Vi si raccontano, come è noto, le varie storie che le diverse posizioni di due mazzi di tarocchi suggeriscono. È vero che-come lo stesso autore ci dice-la suggestione più diretta viene dalle ricerche di studiosi sovietici sulla "cartomanzia come sistema semiotico", ${ }^{3}$ ma i punti di contatto con componenti importanti della tradizione mnemotecnica sono di grande spessore. Si tratta infatti di procedere disponendo nello spazio, secondo sequenze variabili, delle immagini; le storie che ne nascono si legano sia alla capacità che le singole immagini hanno di attivare catene di associazioni, sia all'ordine in cui sono disposte, e quindi alle diverse reti di relazioni che si vengono così creando.

Può apparire allora molto interessante il fatto che, nella fitta rete di rapporti intertestuali in cui si colloca il Castello dei destini incrociati, un posto di grande rilievo spetti all'Orlando furioso. Calvino lo giustifica in relazione all'esigenza di usare come chiave della narrazione-come "fonti topici", diremmo con linguaggio cinquecentesco-i tarocchi dei Visconti:

il riferimento letterario che mi veniva spontaneo era l'Orlando furioso. Anche se le miniature di Bonifacio Bembo precedevano di quasi un secolo il mondo di Ludovico Ariosto, esse potevano ben rappresentare il mondo visuale nel quale la fantasia ariostesca s'era formata. Provai subito a comporre con i tarocchi viscontei sequenze ispirate all'Orlando furioso: $\mathrm{mi}$ fu facile cosi costruire l'incrocio centrale dei racconti del mio "quadrato magico". Intomo, bastava lasciare che prendessero forma altre storie che s'incrociavano tra loro, e ottenni così una specie di cruciverba fatto di figure anziché di lettere in cui per di più ogni sequenza si può leggere nei due sensi. Nel giro d'una settimana, il testo del Castello dei destini incrociati. . . era pronto per essere pubblicato. (125)

Nella rievocazione di Calvino, dunque, si passa immediatamente dalla notazione di carattere storico e culturale a sottolineare l'automatismo e la straordinaria rapidità di funzionamento della macchina narrativa che si mette 
in moto facendo interagire i tarocchi viscontei con l'Orlando furioso. Il paragone con il cruciverba ("fatto di figure anziché di lettere") riporta in primo piano la componente del gioco e, nello stesso tempo, sottolinea come sia essenziale, per la "produzione" delle storie, la dimensione dello spazio. ${ }^{4}$

Può allora essere interessante ricordare che nel '600 Marco Aurelio Severino-un medico napoletano allievo di Campanella, studioso, tra l'altro, della Topica di Giulio Camillo-scrive un'opera in cui sottolinea le profonde affinità tra il gioco degli scacchi e la poesia, e individua proprio nell'Orlando furioso il poema dove tutto ciò diventa pienamente visibile e dove le potenzialità che ne derivano trovano una splendida realizzazione.

Anche dal modo-per certi aspetti stravolto e frantumato-in cui l'arte della memoria ricompare nel nostro tempo ci viene, io credo, un invito a interrogarci in modo nuovo sul suo passato. Questo significa, come accennavo, usare i trattati mnemotecnici come un semplice punto di partenza per ripercorrere la ricca e multiforme sperimentazione che il passato ha compiuto nei territori della memoria.

Numerosi indizi ci spingono ad esempio a pensare che l'arte della memoria non sia servita soltanto a ricordare testi e immagini già esistenti, ma anche a produrre testi e immagini "memorabili", tali, cioè, da essere ricordati con facilità e fedeltà. La tradizione retorica in genere dà alla memoria un posto secondario. Insieme alla actio essa appare come qualcosa che interviene quando il testo è ormai fatto, a facilitare la sua presentazione al pubblico, e quindi la sua efficacia. Possiamo dire che in molti casi, nel Medioevo e nel Rinascimento, non è stato cosi: la memoria pud essere vista come una componente interna alla creazione del testo, insieme alla dispositio e alla elocutio. In un contesto profondamente mutato, nell'era della scrittura e della stampa, la memoria mantiene dunque qualcosa della sua antica dignità, quella che ne faceva una dea, Mnemosyne, la madre delle Muse, la depositaria della conoscenza, oltre che del piacere della poesia. Proprio nell'ottica dell'arte della memoria, del resto, Giordano Bruno mette in risalto l'analogia profonda di procedimenti che accomuna il pittore, il poeta, il filosofo e, appunto, chi pratica la mnemotecnica (123).

Cercare, attraverso i secoli, le tracce di quella che possiamo chiamare l'operatività retorica della memoria pud dare risultati interessanti, pud ad esempio aiutare a capire la ragione e la logica di certe intersezioni fra parole e immagini che altrimenti non verrebbero alla luce, o apparirebbero senza senso. Mi limiterd qui a fare solo qualche esempio, legato alle mie esperienze di ricerca. Particolarmente ricco di prospettive si è mostrato il terreno della predicazione e della letteratura religiosa nel Medioevo e nel Rinascimento. Il testo di un domenicano di fine '300, il Colloquio spirituale di Simone da Cascina, in cui si spiegano i significati riposti nella liturgia della Messa, si è rivelato prezioso: esso $\mathrm{mi}$ ha aperto la porta non solo su di un ricco repertorio di immagini, ma anche su di un preciso codice retorico e culturale, che ne guida la formazione (cfr. Bolzoni, "Il Colloquio"). 
Il testo di Simone, che ci è giunto attraverso un unico codice non iconografato, delinea con le parole una serie di immagini allegoriche che si propongono di collocarsi in modo stabile ed efficace nella mente del destinatario, di influire sul suo intelletto, sulla sua memoria, sulla sua volontà, così da operare nella sua anima un processo di purificazione e trasformazione interiore, che può arrivare fino all'ascesi mistica. Molte delle immagini descritte nel testo di Simone trovano una puntuale corrispondenza in alcune immagini miste (costruite cioè con la pittura e con la scrittura), miniate e dipinte, ampiamente diffuse in Europa a partire dal '200, appartenenti alla tipologia dei Bilderbücher. Basterà ricordare qui la turris sapientiae, o il lignum vitae, o gli schemi che visualizzano le corrispondenze fra vizi, virtù e precetti morali. Ma c'è qualcosa di ancora più interessante, che va al di là della precisa corrispondenza iconografica e investe la logica, i modi stessi della costruzione delle immagini. Le enumerazioni, le antitesi, le metafore sviluppate che caratterizzano il testo di Simone (e di molti altri testi medioevali) risultano costruite con lo stesso codice retorico che guida la produzione dei Bilderbücher. Si tratta in ogni caso di delineare-con la scrittura, con la pittura, o con entrambe-immagini capaci di imprimersi nella memoria così da mettere in moto la catena di associazioni che guida a quel processo di trasformazione interiore di cui si è detto sopra. Se adottiamo questo punto di vista, comprendiamo che le immagini miste dei Bilderbücher funzionano da vere e proprie macchine testuali: esse condensano in sé testi esistenti, danno un ordine retorico a un sistema di conoscenze; esse dunque possono aiutarci a produrre altri testi, possono fornirci la chiave per sviluppare altre catene di associazioni, altre reti di corrispondenze.

Nel ' 400 un predicatore di grande successo come san Bernardino da Siena fa un uso spregiudicato di tutte le risorse dell'arte della memoria. Utilizza una delle vecchie immagini dei Bilderbücher, il serafino, come griglia di un intero ciclo di predicazione. Le penne delle ali del serafino, gli splendori che escono dal suo volto, gli servono per ricordare-e per far ricordare agli "intendenti"-lo schema e le articolazioni giomaliere del quaresimale che egli predica a Firenze nel 1424 (cfr. Bolzoni, "Gedächtniskunst"). Ma è l'intera città, con le sue vie, i suoi edifici e i suoi dipinti, che diventa il teatro della memoria della predicazione di san Bernardino. Per far durare l'efficacia dei suoi insegnamenti al di là della durata effimera della predica, san Bernardino trasforma le pitture cittadine in immagini allegoriche e, insieme, in immagini di memoria delle cose che ha insegnato; in questo modo, anche quando egli sarà lontano, esse faranno rivivere, nella mente delle persone, le parole e concetti che san Bernardino aveva loro affidato.

L'arte della memoria è fra gli strumenti usati creativamente anche da un altro grande trascinatore di masse cittadine: Gerolamo Savonarola. Anche in lui c'è un intreccio profondo fra allegoria e immagini di memoria, ma tutto è finalizzato a rendere credibile, e operante, il suo messaggio profetico. Gli eventi e i personaggi della Bibbia — del Vecchio Testamento in primo luogo- 
diventano, nella sua esegesi, immagini attraverso le quali decifrare il presente e il futuro, immagini di straordinaria efficacia, capaci di far vedere come, dal punto di vista di Dio, il tempo non esiste e come, nella Bibbia, tutto è in tutto. L'interpretazione allegorica della Bibbia produce così immagini profetiche, capaci di parlare alla memoria e alla immaginazione grazie al loro carattere sensibile. Ed è significativo che il Savonarola sia il primo, in Italia, a capire le straordinarie possibilità nuove offerte dalla stampa, e che affidi le sorti della sua battaglia anche al libro, e al libro illustrato in particolare (cfr. Bolzoni, "Oratoria" 1053-57; AA. VV., Immagini).

La letteratura religiosa, fra Tre e Quattrocento, ci testimonia dunque la fertile creatività dell'arte della memoria. Essa interagisce con l'allegoria, e quindi con l'insegnamento morale, con la profezia, con le tecniche di elevazione mistica. Di questo va tenuto conto anche per cogliere in tutto il suo spessore il senso dell'operazione compiuta, nel primo '500, da Giulio Camillo (cfr. Dizionario; Vasoli; Bolzoni, Il teatro; Bologna). In un breve scritto inedito, il De transmutatione, egli rivela il segreto fondamentale che sta alla base del suo teatro della memoria: esso intende essere la guida al compimento della grande opera della trasmutazione, la quale si attua nell'eloquenza, nell'alchimia, nella deificazione, perché riguarda rispettivamente la capacità di trasformare le parole, le cose e l'interiorità dell'uomo. La nostra prima reazione potrebbe essere quella di vedere in queste pagine una delle tante vanterie del Camillo, che gli procurarono una fama un po' equivoca in tutta Europa. Se però teniamo presente quanto si accennava, vediamo che le cose stanno in modo diverso. Nei termini propri del neoplatonismo e dell'ermetismo cinquecentesco, il Camillo ripropone qui un nesso che aveva funzionato per secoli nella pratica della Chiesa, e cioè il nesso fra arte della memoria, costruzione di testi efficaci e trasformazione interiore, ascesi mistica.

Il teatro del Camillo, si diceva, è anche una guida all'eloquenza. Esso promette infatti di fornire la via facile e breve che permette l'imitazione dei modelli esemplari. Il teatro dunque si propone come la macchina capace di garantire, con il suo funzionamento, gli stessi risultati che la tradizionale educazione umanistica prometteva solo come frutto di un lungo lavoro di educazione letteraria. Proprio per questo il teatro del Camillo diventa il punto di riferimento per coloro che, nel secondo '500, fabbricano "alberi", tavole, diagrammi retorici, di coloro che-influenzati dal lullismo e dalla nuova dialettica di Rodolfo Agricola e di Pierre de la Ramé-costruiscono vere e proprie macchine logiche e retoriche capaci di produrre le trame e gli artifici dei testi.

Ma il teatro del Camillo è anche una grande, straordinaria galleria di immagini. Collocate nella fitta trama dei loci del teatro, sono le immagini ad aprire le porte sui tesori dell'eloquenza, minutamente schedati attraverso l'"anatomia" dei testi esemplari: sono le immagini che insieme esibiscono e nascondono, esprimono e celano, il contenuto universale di bellezza e di sapere che è depositato nel teatro. 
Le immagini del teatro del Camillo, costruite usando le risorse di una raffinata cultura ermetica, cabalistica, emblematica e antiquaria, oltre che letteraria, affidate per la loro realizzazione sensibile a grandi pittori, come il Tiziano o Francesco Salviati, costituiscono per noi una specie di sfida a ripensare nella sua complessita la tematica dell'ut pictura poësis nel '500 e, in particolare, i suoi intrecci con l'arte della memoria.

Ci si limiterà qui a qualche acccuno. Francesco Sansovino, il figlio del grande architetto, in un breve scritto sulla retorica spiega il significato delle statue-di Apollo, Mercurio, la Pace, il Sole, Pallade-che il padre ha collocato nella Loggetta di Piazza San Marco a Venezia. Ciascuna di esse appare come l'allegoria delle qualità che fanno grande il potere della repubblica veneziana e proprio per questo, scrive il Sansovino, esse sono un esempio efficace di "luoghi della memoria".

$\mathrm{Nel} 1569$ un architetto molto colto, grande esperto di antiquaria, Pirro Ligorio, disegna 16 scene della vita dell'antico eroe Ippolito, in onore del suo illustre committente, il cardinale Ippolito d'Este. I disegni di Ligorio, infatti, dovranno essere riprodotti su arazzi che a loro volta sono destinati a ornare la splendida villa che il cardinale si è fatto costruire a Tivoli. Le virtù del padrone di casa si riflettono nelle scene esemplari della vita dell'eroe greco omonimo. Spiegando i significati allegorici delle scene da lui disegnate, Pirro Ligorio dice che così egli imita gli antichi, i quali usavano le immagini dei grandi personaggi del passato come "memoria locale".

Il pieno '500 vede il trionfo di un preciso genere retorico: il genere epidittico o dimostrativo, quello che insegna non a decidere e a giudicare, ma soltanto a lodare o a biasimare. Si tratta di un contesto retorico profondamente influenzato dalla seconda sofistica, da quei retori cioè che fra l'altro avevano coltivato il gusto per una intensa visualizzazione e per una erudizione raffinata. I casi da noi citati sono pienamente ascrivibili al genere epidittico: la Loggetta del Sansovino è un panegirico visualizzato della Serenissima Repubblica di Venezia, e Villa d'Este, con il suo complesso sistema decorativo, è un panegirico visualizzato del cardinale Ippolito d'Este. L'arte della memoria, dunque, interagisce con le immagini allegoriche ed è una componente importante del nuovo codice retorico.

Il modello che regola le immagini collocate nelle piazze e nelle ville è lo stesso che, in ambito religioso, guida nel primo '600 la costruzione dei livres-galéries dei Gesuiti. Questi libri, splendidamente illustrati, funzionano, come ha mostrato Marc Fumaroli, da veri e propri palazzi della memoria e segnano un percorso di elevazione a Dio in cui le immagini sensibili servono da "sceaux intérieurs de la mémoire chrétienne".

Tutto questo comporta il pieno trionfo retorico di quella concezione spaziale della letteratura che la scrittura aveva introdotto e che la stampa aveva sviluppato. Il modello dell'edificio si sovrappone a quello del testo: poemi e orazioni vengono percepiti come palazzi, come un insieme di luoghi architettonici. Il Sansovino ad esempio paragona il proemio di un discorso 
all'ingresso di un palazzo (4v); Galileo, per esprimere la sua ammirazione per l'Ariosto e il suo disprezzo per il Tasso, paragona l'Orlando furioso a una splendida galleria regia e la Gerusalemme liberata a uno "studiolo" squallido e pretenzioso (130-31). Luoghi fisici, luoghi della memoria, luoghi retorici, tendono dunque a sovrapporsi, con un complesso gioco di rinvii e di diffrazione.

Si tratta di un codice così fortemente suggestivo che, nel momento in cui viene negato, impone ancora una volta le sue immagini topiche, anche se ormai ridotte a fantasmi. "Non ha la memoria una galeria muta di pitture variabili-scrive il Ciampoli, il poeta segretario di papa Urbano VIII e amico di Galileo-non vi si stampano, non ci si fissano. È una popolazione di simolacri viventi" (348-49). ${ }^{5}$

$\mathrm{Ma}$, naturalmente, la partita non era affatto chiusa una volta per tutte. Allo stesso modo la satira feroce con cui, nel '700, Swift descrive la macchina girevole che serve per scrivere tutti i libri e che Gulliver vede nell'Accademia di Lagado (233 sgg.), non distrugge, come abbiamo visto, l'interesse per la sperimentazione di procedimenti automatici e combinatori nella scrittura letteraria. Certo si tratta di suggestioni che tornano di volta in volta sotto forme diverse, ma l'arte della memoria si è assicurata una sopravvivenza secolare proprio grazie alla sua capacità di trasformarsi. Essa è per eccellenza l'arte della metamorfosi: ce lo insegna Giulio Camillo, istrionico gran maestro di memoria, e gli possiamo credere.

Università di Pisa

\section{NOTE}

1 Per la ormaj ricca tradizione di studi relativa al passaggio dalla oralità alla scrittura, mi limito a rinviare a Ong.

2 La mostra si è tenuta a Firenze dal 23 marzo al 26 giugno 1989 e a Parigi dal 9 aprile 1990 al 2 gennaio 1991.

3 Cfr. Lekomceva e Uspenskij. Sul romanzo di Calvino, mi limito a rinviare a Corti.

4 Cruciverba e macchina narrativa si sovrappongono anche in altri autori contemporanei; mi limito qui a ricordare Georges Perec, amico di Calvino e, come lui, legato al gruppo dell'Oulipo.

5 G. Ciampoli. Prose. Roma: 1649; citato da Raimondi, 329.

\section{OPERE CITATE}

AA. VV. Mnemosyne. Formen und Funktionen der kulturellen Erinnerung. Frankfurt am Mein: Fischer, 1991.

AA. VV. Immagini e azione riformatrice: le xilografie degli incunaboli savonaroliani nella Biblioteca Nazionale di Firenze. Firenze: Alinari, 1985.

Benjamin, Walter. "Baudelaire e Parigi". Angelus Novus. Saggi e frammenti. Torino: Einaudi, 1976. 85-154.

Bologna, Corrado. "Il Theatro segreto di Giulio Camillo: l'Urtext ritrovato". Venezia Cinquecento 1.2 (1991): 217-71. 
Bolzoni, Lina. "Il Colloquio spirituale di Simone da Cascina. Note su allegoria e immagini della memoria". Rivista di letteratura italiana 3.1 (1985): 9-65.

"Gedächtniskunst und allegorisches Bilder. Theorie und Praxis der ars memorativa in Literatur und Bildender Kunst Italiens zwischen dem 14. und 16. Jahrhundert". AA. VV. Mnemosyne. 155-60.

"Il gioco delle immagini. L'arte della memoria dalle origini al Seicento". La

fabbrica del pensiero. Dall'arte della memoria alle neuroscienze. Catalogo della mostra. Firenze, Forte di Belvedere, 23 marzo-26 giugno 1989. Milano: Electa, 1989. 16-65.

. "Oratoria e prediche". Letteratura italiana. A cura di A. Asor Rosa. Vol. 3.2.

Torino: Einaudi, 1984. 1041-74.

Il teatro della memoria. Studi su Giulio Camillo. Padova: Liviana, 1984.

Bruno, Giordano. "Explicatio triginta sigillorum ad omnium scientiarum et artium inventionem, dispositionem, et memoriam". Opera latine conscripta. Vol. 2. Eds. F. Tocco e E. Vitelli. Stoccarda: Bad Cannstatt, 1962. [Firenze: 1890.]

Calvino, Italo. Il castello dei destini incrociati. Torino: Einaudi, 1973.

Camillo, Giulio. L'idea del theatro. Ed. L. Bolzoni. Palermo: Sellerio, 1991.

Carruthers, Mary. The Book of Memory: A Study of Memory in Medieval Culture. Cambridge and New York: Cambridge UP, 1990.

Corti, Maria. "Le jeu comme génération du texte". Semiotica 7 (1973): 33-48.

Della Porta, Giovan Battista. Ars reminiscendi. Napoli: Giovan Battista Sottile, 1602.

Dizionario biografico degli italiani. Ed. G. Stabile. Roma: Istituto della Enciclopedia italiana, 1973.

Fumaroli, Marc. L'age de l'éloquence. Rhétorique et "res literaria" de la Renaissance au seuil de l'époque classique. Genève: Droz, 1980.

Galilei, Galileo. "Considerazioni al Tasso". Le opere. Vol. 15. Firenze: Società Editrice Fiorentina, 1856.

Gedächnistkunst: Raum-Bild-Schrift. Studien zur Mnemotechnik. Eds. A. Haverkamp und R. Lachmann. Frankfurt am Mein: Suhrkamp, 1991.

Lekomceva, M.I. e B.A. Uspenskij. "La cartomanzia come sistema semiotico". I sistemi di segni e lo strutturalismo sovietico. Eds. R. Faccani e U. Eco. Milano: Bompiani, 1969. 243-48.

Ligorio, Pirro. "Vita di Virbio, detto altrimenti Hippolito figlio di Theseo descritta e dissegnata con immitatione dell' antico in siedici historie". David R. Coffin. The Villa d'Este at Tivoli. Princeton: Princeton UP, 1960.

Lurija, Alexander A. Viaggio nella mente di un uomo che non dimenticava nulla. Roma: Armando, 1979.

Massin. La lettre et l'image. La figuration dans l'alphabet latin du huitième siècle d nos jours. Parigi: Gallimard, 1970.

Ong, Walter J. Oralitd e scrittura. Le tecnologie della parola. Bologna: Il Mulino, 1982.

Raimondi, Ezio. Letteratura barocca. Studi sul Seicento italiano. Firenze: Olschki, 1961.

Rossi, Paolo. "Clavis universalis". Arti della memoria e logica combinatoria da Lullo a Leibniz.

Milano: Ricciardi, 1960.

Sacks, Oliver. L'uomo che scambid sua moglie per un cappello. Milano: Adelphi, 1986.

Sansovino, Francesco. In materia dell'arte libri tre ne' quali si contien l'ordine delle cose che si ricercano nell'oratore. Venezia: Sansovino, 1561.

Severino, Marco A. La filosofia overo il perché degli scacchi. Napoli: Antonio Bulifon, 1690.

Simone Da Cascina. Colloquio spirituale. Ed. F. Dalla Riva. Firenze: Olschki, 1982.

Swift, Jonathan. "I viaggi di Gulliver". Opere. Milano: Mondadori, 1983.

Vasoli, Cesare. I miti e gli astri. Napoli: Guida, 1977.

Yates, Francis A. L'arte della memoria. Torino: Einaudi, 1972. 